\title{
Simulation of fluid-structure interaction with the interface artificial compressibility method
}

\author{
Joris Degroote $^{1}$, Abigail Swillens ${ }^{2}$, Peter Bruggeman ${ }^{3}$, \\ Robby Haelterman ${ }^{4}$, Patrick Segers ${ }^{2}$, Jan Vierendeels ${ }^{1}$ \\ ${ }^{1}$ Ghent University, Department of Flow, Heat and Combustion Mechanics \\ Sint-Pietersnieuwstraat 41, B-9000 Ghent, Belgium \\ ${ }^{2}$ Ghent University, Institute Biomedical Technology \\ De Pintelaan 185, B-9000 Ghent, Belgium \\ ${ }^{3}$ Ghent University, Department of Applied Physics \\ Jozef Plateaustraat 22, B-9000 Ghent, Belgium \\ ${ }^{4}$ Royal Military Academy, Department of Mathematics (MWMW) \\ Renaissancelaan 30, B-1000 Brussels, Belgium
}

\begin{abstract}
Partitioned fluid-structure interaction simulations of the arterial system are difficult due to the incompressibility of the fluid and the shape of the domain. The interface artificial compressibility method (IAC) mitigates the incompressibility constraint by adding a source term to the continuity equation in the fluid domain adjacent to the fluid-structure interface. This source term imitates the effect of the structure's displacement as a result of the fluid pressure and disappears when the coupling iterations have converged. The IAC method requires a small modification of the flow solver but not of the black box structural solver and it outperforms a partitioned quasi-Newton coupling of two black box solvers in a simulation of a carotid bifurcation.
\end{abstract}

Keywords: fluid-structure interaction, interface artificial compressibility, interface block quasi-Newton, carotid bifurcation.

\section{Introduction}

Numerical simulation of various biomedical topics has gained in interest over the last decade [1]. In particular, fluid-structure interaction (FSI) in the arterial system is studied with the aim of improving artificial heart valves [2, 3, 4] and predicting 
the rupture of aneurysms or the outcome of surgical procedures [5, 6]. In multiscale simulations, a detailed three-dimensional simulation of the area of interest is coupled with one-dimensional models which act as boundary conditions and represent the rest of the cardiovascular system [7]. Moreover, patient-specific data are used for both material models and geometry to increase the fidelity of the simulations $[8,9]$.

FSI simulations in general can be solved in a monolithic or partitioned way. The monolithic approach is to solve the flow equations and the structural equations simultaneously [10]. In a partitioned simulation, the governing equations in the flow domain and in the structural domain are solved with a separate flow solver and structural solver so coupling iterations have to be performed between these solvers and the exchange of information is limited to the fluid-structure interface. The main advantage of the monolithic approach is that no coupling iterations within the time step are required. On the other hand, existing flow solvers and structural solvers can be employed in a partitioned simulation, reducing software development efforts and improving manageability.

It is common to use a Dirichlet-Neumann partitioning for FSI simulations which means that the flow problem is solved for a given displacement of the fluid-structure interface and that a stress boundary condition is applied on the wet boundary of the structural problem [11]. This partitioning is chosen because most flow solvers cannot calculate both the flow variables and the mesh position for a given stress on a boundary.

In a block Gauss-Seidel scheme with Dirichlet-Neumann partitioning, the flow problem is solved for a given position of the fluid-structure interface, followed by a calculation of the deformation of the structure due to the fluid stress on the fluidstructure interface and subsequently iterations are performed until convergence is obtained. The convergence of this simple scheme is however very slow for FSI simulations with flexible structures and incompressible fluids, if it converges at all, but it can be accelerated by using Aitken relaxation [12]. Other techniques to accelerate the convergence include the interface block quasi-Newton technique with least-squares reduced-order models (IBQN-LS) $[13,14]$ and the interface quasi-Newton technique with inverse Jacobian from a least-squares model (IQNILS) [15] which provide superior convergence of the coupling iterations between two black-box solvers in comparison with relaxation methods.

Block Newton-Raphson methods can also be used in both monolithic [16, 17, 10] and partitioned [18, 19, 20] solution techniques for FSI problems. These methods solve the nonlinear flow equations and the structural equations for the variables in the entire fluid and solid domain with the Newton-Raphson method. The linear system within the Newton-Raphson iteration can be solved without knowledge of the Jacobian with a matrix-free Krylov solver, using a finite-difference approximation of the Jacobian-vector product [10]. Both monolithic and parti- 
tioned Newton-Raphson methods for FSI problems are described by Tezduyar et al. [21, 22].

To calculate the flow in a deforming domain, the flow equations are frequently written in arbitrary Lagrangian-Eulerian formulation and solved on a moving mesh. The deformation of the fluid mesh due to a displacement of the fluidstructure boundary can be calculated with a structural analogy. If the mesh becomes too distorted, remeshing has to be performed. An alternative is the immersed boundary method of Peskin [23, 24] which is also common in FSI for biomedical problems. In this method, the flow equations are solved on an Eulerian mesh and the effect of the immersed boundary on the fluid is given by a force density function. A disadvantage of the latter method and other fictitious domain methods is that it is harder to calculate stresses on the fluid-structure interface accurately, which can be mitigated by adaptive meshing [25]. However, fictitious domain methods are practical in heart valve simulations where the fluid domain is divided in separate regions by the motion of the structure [26].

Causin et al. [11] demonstrated the difficulties of partitioned FSI with incompressible fluids and flexible structures by rewriting the flow equations as an added-mass operator in the structural equations. Substitution of a linear reducedphysics model of the flow into the structural equations approximates the addedmass operator and thus it accelerates the convergence of the coupling iterations [27]. In [28], block Gauss-Seidel iterations between the flow solver and the structural solver and the effect of substituting a linearized model for the structure in the flow equations are analyzed with Fourier error analysis. This analysis on a onedimensional flexible tube shows that the perturbations of the interface's position with a low wavenumber are most unstable due to the incompressibility constraint of the fluid. Especially the substitution of the linearized structural model in the continuity equation of the flow problem proved to stabilize the coupling iterations.

This substitution term in the continuity equation of the flow problem can be interpreted as compressibility but the term disappears completely when the block Gauss-Seidel iterations have converged such that the solution of the incompressible flow equations is found. Hence, this technique was named artificial compressibility method and it has previously been applied to two- and three-dimensional problems with simple geometries [29, 30, 31, 32]. The artificial compressibility method has been improved by limiting the compressibility to the fluid cells adjacent to the fluid-structure interface, which is called interface artificial compressibility (IAC), and it has been demonstrated briefly how the compressibility coefficients can be obtained from a black-box structural solver [33, 34]. Due to the addition of the substitution term, the IAC method cannot be combined with the above mentioned acceleration techniques for block Gauss-Seidel iterations like the IBQN-LS method.

The concept of the artificial compressibility method for FSI is similar to the 
artificial compressibility method of Chorin [35] and Témam [36] who introduced compressibility in pseudo-time to calculate steady flow of incompressible fluids. This concept was later extended to unsteady simulations by Peyret [37] and Merkle and Athavale [38] who used a calculation in pseudo-time within every time step. In the artificial compressibility method for FSI, the iterations between the flow solver and the structural solver within the time step can also be considered as pseudo-time. It will be shown that if the artificial compressibility is only used at the interface, this corresponds to an implicit treatment of the boundary displacement while the flow equations are solved.

In this paper, the IAC method is applied to an FSI simulation of a patientspecific carotid bifurcation geometry which is a much larger and more complex problem than those previously studied with IAC. The procedure to calculate the compressibility coefficients for a black-box structural solver is explained in detail and the performance of the IAC method is compared with interface block quasiNewton.

Several studies on bifurcations of arteries have been performed before with other methods. Some were limited to flow calculations [39] or prescribed motion of the artery wall [40] while Scotti et al. [41] performed FSI simulations of patient-specific abdominal aortic aneurysms with an iliac bifurcation and localized intraluminal thrombus using the monolithic solver of ADINA (Adina R\&D, Inc., Watertown, MA, U.S.A.) [17]. Perktold et al. [42] simulated a carotid artery bifurcation with block Gauss-Seidel iterations between the flow solver and the structural solver and Yang et al. [43] studied the pulmonary arterial bifurcation with the same technique [44].

The remainder of this paper is outlined as follows. The governing equations for the flow and the structure are given in section 2 and subsequently the IAC and IBQN-LS method are explained in section 3. In section 4, both coupling techniques are verified by performing a simulation of a three-dimensional straight elastic tube and finally the results for the carotid bifurcation are given.

\section{Governing equations}

In this section, the governing equations for the fluid flow and the structure are outlined briefly. The fluid domain and structural domain are indicated as $\Omega_{f}$ and $\Omega_{s}$, respectively, and their boundaries as $\Gamma_{f}$ and $\Gamma_{s}$. The fluid-structure interface $\Gamma_{i}=\Gamma_{f} \cap \Gamma_{s}$ is the common boundary of these domains. 


\subsection{Flow equations}

The unsteady flow of an incompressible, isothermal fluid is governed by the conservation of mass and the Navier-Stokes equations, given by

$$
\begin{gathered}
\nabla \cdot \boldsymbol{v}=0 \\
\rho_{f} \frac{\partial \boldsymbol{v}}{\partial t}+\rho_{f} \nabla \cdot(\boldsymbol{v} \boldsymbol{v})-\nabla \cdot \boldsymbol{\tau}_{f}=\boldsymbol{f}_{f}
\end{gathered}
$$

for $\boldsymbol{x} \in \Omega_{f}$. The flow velocity is denoted by $\boldsymbol{v}, \rho_{f}$ is the fluid density, $t$ the time and $\boldsymbol{f}_{f}$ the body forces per unit of volume on the fluid. For a Newtonian fluid, the stress tensor is defined as $\boldsymbol{\tau}_{f}=-p \boldsymbol{I}+2 \mu \boldsymbol{\gamma}$ with $\boldsymbol{\gamma}=\frac{1}{2}\left(\nabla \boldsymbol{v}+(\nabla \boldsymbol{v})^{\mathrm{T}}\right)$ the rate of strain tensor and $\mu$ the dynamic fluid viscosity. Using the Dirichlet-Neumann partitioning, the flow problem is subject to the kinematic boundary condition

$$
\boldsymbol{v}=\dot{\boldsymbol{u}}
$$

for $\boldsymbol{x} \in \Gamma_{i}$ with $\boldsymbol{u}$ the structural displacement and appropriate conditions on the remainder of the boundary $\Gamma_{f} \backslash \Gamma_{i}$.

In FSI calculations, these equations have to be solved on a moving mesh due to the deformation of the structure and therefore they are discretized in the arbitrary Lagrangian-Eulerian formulation. For the discrete equations in a control volume to be conservative in time, the volume swept by the control volume's boundaries must be calculated in such a way that it is consistent with the time discretization of the change of its volume. Therefore, every discretization has its own requirement with respect to a consistent calculation of the time-dependent geometric quantities which is commonly referred to as the geometric conservation law [45].

Equations (1), integrated over an arbitrary, deforming control volume $V$ are given by

$$
\begin{gathered}
\frac{\partial V}{\partial t}+\int_{\partial V}\left(\boldsymbol{v}-\boldsymbol{v}_{b}\right) \cdot \mathrm{d} \boldsymbol{A}=0 \\
\rho_{f} \frac{\partial}{\partial t} \int_{V} \boldsymbol{v} \mathrm{d} V+\rho_{f} \int_{\partial V} \boldsymbol{v}\left(\boldsymbol{v}-\boldsymbol{v}_{b}\right) \cdot \mathrm{d} \boldsymbol{A}-\int_{\partial V} \boldsymbol{\tau}_{f} \cdot \mathrm{d} \boldsymbol{A}=\int_{V} \boldsymbol{f}_{f} \mathrm{~d} V
\end{gathered}
$$

with $\partial V$ the the control volume's boundary, $\boldsymbol{A}$ the area vector pointing outward and $\boldsymbol{v}_{b}$ the velocity of the control volume's boundary.

The process of solving the flow equations (3) for a given position of the fluidstructure interface is indicated by the function

$$
T=\mathcal{F}(X)
$$

which is referred to as the flow solver and which concisely represents several operations. The discretized position $X \in \mathbb{R}^{N}$ of the entire fluid-structure interface 
$\Gamma_{i}$ is passed to the flow code and the grid of the fluid domain adjacent to the interface is adapted accordingly. Subsequently, the flow equations are solved for the fluid state and the distribution of the fluid stress on $\Gamma_{i}$ is returned as $T \in \mathbb{R}^{M}$. Both $X$ and $T$ are at the new time level $t^{n+1}$, as is $\mathcal{F}$ such that the boundary conditions and other settings of $\mathcal{F}$ are also adapted to the new time level. In the remainder of this paper, all values and functions are at the new time level $n+1$, unless indicated otherwise with a superscript. A subscript indicates the coupling iteration within the time step.

\subsection{Structural equations}

The deformation of the structure is determined by the conservation of momentum

$$
\rho_{s} \frac{\partial^{2} \boldsymbol{u}}{\partial t^{2}}-\nabla \cdot \boldsymbol{\tau}_{s}=\boldsymbol{f}_{s}
$$

for $\boldsymbol{x} \in \Omega_{s}$ with $\rho_{s}$ the structural density and $\boldsymbol{f}_{s}$ the body force per unit volume on the structure. The relation between the stress tensor $\tau_{s}$ and the strains is given by the constitutive equation of the material. Again using the Dirichlet-Neumann partitioning, the structural problem is subject to the dynamic boundary condition

$$
\boldsymbol{\tau}_{s} \cdot \boldsymbol{n}_{s}=-\boldsymbol{\tau}_{f} \cdot \boldsymbol{n}_{f}
$$

for $\boldsymbol{x} \in \Gamma_{i}$ with $\boldsymbol{n}_{s}$ and $\boldsymbol{n}_{f}$ the unit normal vectors pointing outwards of $\Omega_{s}$ and $\Omega_{f}$, respectively, and suitable boundary conditions on $\Gamma_{s} \backslash \Gamma_{i}$.

The structural solver is represented by the function

$$
X=\mathcal{S}(T),
$$

which indicates that the fluid stress on $\Gamma_{i}$ is passed to the structural code which then solves the entire structural problem and returns the new position of the fluidstructure interface $\Gamma_{i}$. With these definitions, the coupled FSI problem is to find $X$ (and $T$ ) such that

$$
X=\mathcal{S} \circ \mathcal{F}(X) \quad \text { or } \quad R(X)=\mathcal{S} \circ \mathcal{F}(X)-X=0
$$

with $R$ the residual of the coupled problem.

If the flow problem and the structural problem are not discretized in the same way on the fluid-structure interface, an interpolation between the variables of both solvers is required. In this paper, it is assumed that this interpolation is included in one of the solvers if necessary. An overview of interpolation techniques can be found in [46, 47]. When different time discretizations are used by both solvers, it is possible that spurious oscillations in time are present in the acceleration of the fluid and the structure and thus also in the stresses on the interface [48]. 


\section{Coupling techniques}

In partitioned fluid-structure interaction simulations with an incompressible fluid and a structure of comparable density, coupling iterations between the flow problem and the structural problem have to be performed to obtain the coupled solution $[11,49]$. The block Gauss-Seidel scheme mentioned in the introduction is an implicit coupling technique as the equilibrium between the flow and the structure is enforced in every time step by performing coupling iterations between the flow solver and structural solver. However, the coupling iterations themselves are explicit because the interface's position is not adapted during the solution of the flow problem and the stress on the interface does not change while the structural problem is solved. Consequently, the block Gauss-Seidel scheme converges slowly, if at all.

To accelerate the convergence of the coupling iterations, some degree of implicitness or relaxation has to be added during the solution of the flow problem and structural problem. The IAC method introduces implicitness through a source term in the mass conservation of the flow problem with a similar effect on the flow field as the displacement of the fluid-structure interface due to the pressure. On the other hand, the IBQN-LS method creates a least-squares reduced-order model of the black box flow solver and structural solver. The coupled solution of both models can be calculated because their Jacobians with respect to all variables are known.

Both coupling techniques begin a time step with an extrapolation of the interface's position

$$
X_{0}=\frac{5}{2}\left(X^{n}\right)-2\left(X^{n-1}\right)+\frac{1}{2}\left(X^{n-2}\right),
$$

based on the previous time steps. Lower order extrapolations are used for the first two time steps. The residual of the FSI problem is calculated as

$$
R_{k+1}=X_{k+1}-X_{k}
$$

and the coupling iterations in a time step have converged when $\left\|R_{k}\right\|_{2} \leq \epsilon_{o}$ with $\epsilon_{o}$ a fraction of $\left\|R_{1}\right\|_{2}$.

\subsection{Interface artificial compressibility (IAC)}

Due to the incompressibility of the fluid, a perturbation of the interface's position with a low wavenumber accelerates most of the fluid and consequently large pressure gradients arise in the fluid. The resulting stress on the interface frequently causes an even larger displacement of the structure and thus leads to divergence of the iterations. This instability is reduced by adding artificial compressibility in a way that mimics the displacement of the structure [28]. 
To determine the suitable amount of compressibility, the deformation of the structure in the first time step due to two different pressure distributions on the fluid-structure interface is calculated with the black box structural solver.

$$
\begin{aligned}
& X_{a}^{1}=\mathcal{S}\left(T_{a}^{1}\right) \\
& X_{b}^{1}=\mathcal{S}\left(T_{b}^{1}\right)
\end{aligned}
$$

In this paper, $T_{a}^{1}$ and $T_{b}^{1}$ are a constant pressure on the entire interface but a varying pressure is also possible. The pressures $p_{a}^{1}$ and $p_{b}^{1}$ that have been used are estimations for the lowest and highest pressure during the entire simulation but the method is robust with respect to this choice because it also performs well with only a small difference between $p_{a}^{1}$ and $p_{b}^{1}$ as will be demonstrated in Section 4.1. The calculations with the structural solver in equations (11) are performed in the same way as a normal time step, with the same time step and the corresponding inertia forces.

The positions $X_{a}^{1}$ and $X_{b}^{1}$ are subsequently transferred to the flow solver which results in two different deformations of the control volumes adjacent to the fluidstructure interface. The volume $\Delta v o l$ swept by the fluid-structure interface as the interface is displaced from $X_{a}^{1}$ to $X_{b}^{1}$ is calculated for every control volume adjacent to the fluid-structure interface as shown in figure 1 and the artificial compressibility coefficient $c$ is then calculated as

$$
c^{2}=\frac{p_{b}-p_{a}}{\Delta v o l} \frac{v o l}{\rho}
$$

with $v o l$ the volume of the control volume and $\Delta t$ the time step. Equation (12) is similar to the Bramwell-Hill equation for the wave speed $c_{B H}$ in a straight elastic tube with cross sectional area $S$

$$
c_{B H}^{2}=\frac{\mathrm{d} p}{\mathrm{~d} S} \frac{S}{\rho}
$$

and the latter is a more general form of the Moens-Korteweg equation for small deformations of thin-walled tubes.

The compressibility of the fluid near the fluid-structure interface is obtained by adding a source term in the continuity equation

$$
\int_{V_{k}} \frac{1}{\rho c^{2}} \frac{p_{k+1}-p_{k}}{\Delta t} \mathrm{~d} V+\frac{\partial V_{k}}{\partial t}+\int_{\partial V_{k}}\left(\boldsymbol{v}_{k+1}-\boldsymbol{v}_{b, k}\right) \cdot \mathrm{d} \boldsymbol{A}=0
$$

for the control volumes adjacent to the fluid-structure interface. The flow equations are solved for $p_{k+1}$ and $\boldsymbol{v}_{k+1}$ and the additional source term is treated implicitly. When the coupling iterations converge, $p_{k+1}$ is equal to $p_{k}$ such that the 
source term disappears and consequently the solution of the time step corresponds with an incompressible fluid. The flow solver with the artificial compressibility is further indicated as $\tilde{\mathcal{F}}$ and the block Gauss-Seidel scheme with artificial compressibility is shown in algorithm 1.

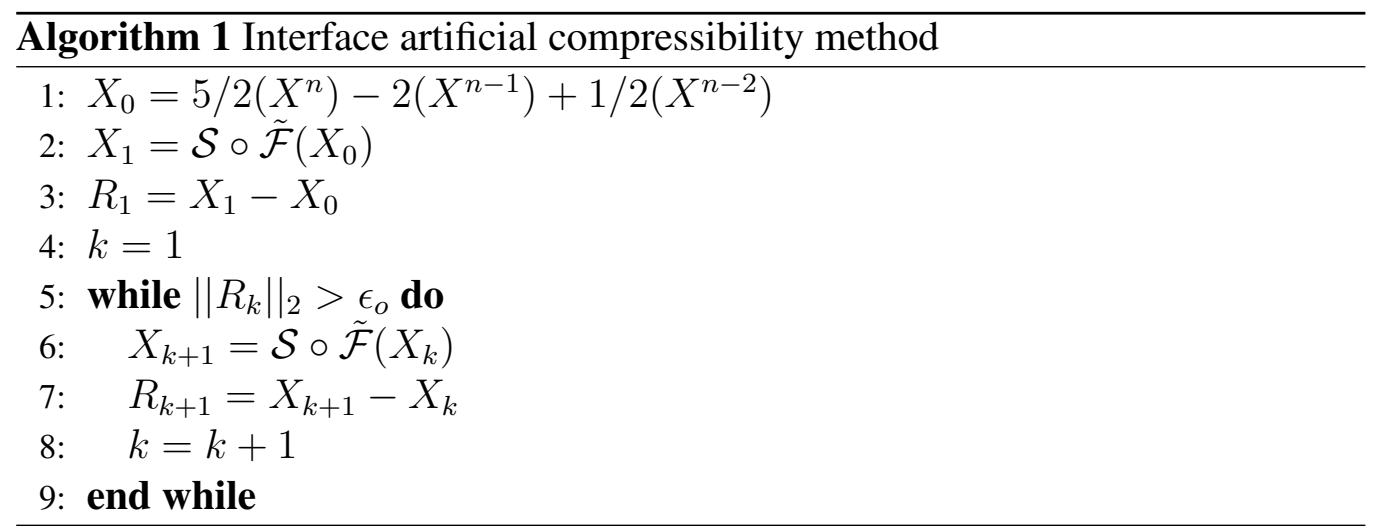

Substitution of equation (12) in equation (14) gives

$$
\frac{\Delta v o l}{p_{b}-p_{a}} \frac{p_{k+1}-p_{k}}{\Delta t}+\frac{\partial V_{k}}{\partial t}+\int_{\partial V_{k}}\left(\boldsymbol{v}_{k+1}-\boldsymbol{v}_{b, k}\right) \cdot \mathrm{d} \boldsymbol{A}=0 .
$$

The first term in the previous equation thus represents a linear approximation for

$$
\frac{V_{k+1}-V_{k}}{\Delta t} .
$$

The similarity with artificial compressibility methods for the simulation of incompressible flows is that the different coupling iterations can be conceived as steps in a pseudo-time. From that point of view, equation (16) is a time derivative in the pseudo-time (with pseudo-time step equal to $\Delta t$ ) which is added to the continuity equation.

If a first-order differencing scheme is used for the second term in equation (15)

$$
\frac{\partial V_{k}}{\partial t} \approx \frac{V_{k}-V^{n}}{\Delta t}
$$

the first two terms of this equation become

$$
\frac{V_{k+1}-V_{k}}{\Delta t}+\frac{V_{k}-V^{n}}{\Delta t}=\frac{V_{k+1}-V^{n}}{\Delta t} .
$$

Consequently, the continuity equation with the IAC term is given by

$$
\frac{\partial V_{k+1}}{\partial t}+\int_{\partial V_{k}}\left(\boldsymbol{v}_{k+1}-\boldsymbol{v}_{b, k}\right) \cdot \mathrm{d} \boldsymbol{A}=0 .
$$


such that the time derivative of $V$ due to the displacement of the interface is now treated implicitly during the solution of the flow equations, albeit in an approximate, linearized way.

If the pressure in a control volume next to the interface changes, the source term mimics how much the structure will move due to that change and it causes a modification of the velocity field such that the velocity already corresponds with the approximated position of the structure. The fluid mass that will be displaced by the motion of the structure is temporarily stored in the pressure field of the control volumes adjacent to the interface as can be seen in equation (19).

Equation (19) also demonstrates that the IAC source term only has to be added to the continuity equation of the control volumes adjacent to the interface. Only the cells next to the interface can take into account the position of the interface in an implicit way. The fluid mass that is stored in the IAC source term will be replaced by real fluid mass in the next coupling iteration once the structural solver has calculated the new position of the interface. For the other control volumes which are not adjacent to the interface, it consequently seems like the interface is already on the approximated position. It is thus important to limit the artificial compressibility to the control volumes adjacent to the fluid-structure interface. If compressibility is added in the entire fluid domain [29, 30, 31, 32], the difference in mass is distributed over the entire fluid domain while the change in volume occurs only at the interface.

The coefficients $c$ are calculated once at the beginning of the unsteady simulation and the same coefficients are used for all time steps. However, these linearized relations between the displacement of the elements on the interface and the pressure are only valid in some range around the pressure for which they have been obtained. If the pressure at the interface changes significantly during the simulation (e.g. due to time-varying boundary conditions), it is possible that new linearization in the neighbourhood of the new pressure have to be calculated.

\subsection{Interface block quasi-Newton (IBQN-LS)}

The interface block quasi-Newton method with two least-squares reduced-order models is explained in detail in [14] and is outlined below for completeness.

In block Gauss-Seidel iterations, the flow problem is solved for different positions of the fluid-structure interface in consecutive iterations. For each of those positions, the flow solver calculates the corresponding stress distribution on the interface. With this sequence of interface positions and the corresponding stress distributions, a least-squares approximation for the Jacobian of $\mathcal{F}$ is calculated. In every subsequent coupling iteration, the flow solver $\mathcal{F}$ calculates the stress distribution on the interface for another position of the interface and this information is used to improve the approximate Jacobian. With the approximate Jacobian of 
the flow solver, the change of the stress distribution on the interface due to a displacement of the interface can be predicted.

The same procedure is followed for the structural solver to obtain an approximate Jacobian for $\mathcal{S}$ such that also the change of the interface's position due to a change of the stress distribution can be estimated. With the approximate Jacobians of $\mathcal{F}$ and $\mathcal{S}$, the coupled solution of the flow solver and the structural solver can be calculated in an approximate but nevertheless implicit way by solving a linear system with as dimension the number of degrees of freedom on the interface. As the accuracy of the least-squares Jacobian improves during the coupling iterations, also the coupled solution becomes more accurate.

The algorithm described in [14] has been extended by reusing the information from the previous $r$ time steps to improve the least-squares estimation for the Jacobians. Moreover, the least-squares problems are solved with QR-decomposition instead of with the normal equations [50] and the above-mentioned linear systems are solved with a matrix-free version of the generalized conjugate residual method [51].

The IBQN-LS method couples two black-box solvers whereas a source term has to be added to the continuity equation in the IAC method. However, the latter is possible in some flow solvers by means of user-defined functions without access to the source code.

\section{Results}

In this section, the implementation of both coupling techniques is verified by simulating the pressure wave propagation in a straight cylinder followed by the simulation with the geometry of a carotid bifurcation. The examples presented below are confined flow problems but both the IAC method and the IBQN-LS method have also been applied successfully to immersed bodies. A simulation of the oscillating beam attached to a rigid cylinder in a laminar flow from the benchmark of Turek et al.[10] results in the same conclusion about the coupling techniques as the examples below (not presented).

\subsection{Tube}

The IAC method and the IBQN-LS method have both been used to simulate a straight elastic tube with inner radius $0.005 \mathrm{~m}$, length $0.050 \mathrm{~m}$ and wall thickness $0.001 \mathrm{~m}$ as described by Fernandez et al. [52] among others [7, 27]. The solid is linear elastic with a Young modulus of $3 \cdot 10^{5} \mathrm{~N} / \mathrm{m}^{2}$ and a Poisson ratio of 0.3 and it has a density of $1200 \mathrm{~kg} / \mathrm{m}^{3}$. The structure is clamped at both ends. The viscosity of the fluid is $0.003 \mathrm{~Pa} \cdot \mathrm{s}$ and its density is $1000 \mathrm{~kg} / \mathrm{m}^{3}$. At the inlet of the tube, a 
pressure of $1333.2 \mathrm{~Pa}$ is applied during $0.003 \mathrm{~s}$ and $0 \mathrm{~Pa}$ afterwards. At the outlet a pressure of $0 \mathrm{~Pa}$ is imposed and the fluid-structure interface is a no-slip boundary. The wave propagation is followed during $0.01 \mathrm{~s}$ with time steps of $0.0001 \mathrm{~s}$.

All simulations have been performed with the flow solver Fluent (Ansys, Inc.,Lebanon, NH, U.S.A) and structural solver Abaqus (Simulia, Inc., Providence, RI, U.S.A.). The finite volume flow solver uses second order discretization for the pressure and second order upwind for the momentum and solves the flow equations for the pressure and velocity in a coupled way with first order time accuracy. The mesh of the fluid domain is adapted to the position of the fluid-structure interface with a spring analogy. The finite element structural solver uses implicit time integration of quadrilateral shell elements with 8 nodes and takes into account the geometric nonlinearities due to the large deformation of the structure. The fluid and structure meshes match each other at the interface.

The mesh for the flow calculation consists of 9282 cells and the mesh on the fluid-structure interface of the fluid model with 1632 rectangular elements has been exported to obtain the mesh for the structural calculation. The coupling algorithm is executed on one core, the flow solver on three cores and the structural solver on two cores of a dedicated machine with two Intel Xeon 5160 dual-core processors.

The pressure contours on the fluid-structure interface in figure 2 show the wave propagation in the straight elastic tube and they compare well with those in [52]. Figure 3 demonstrates that the rate of change of the tube's volume $V$ corresponds excellently with the net volumetric influx throughout the simulation with the IAC method and also with the net volumetric flux in the simulation with the IBQN-LS method. The compressibility has thus disappeared completely once the coupling iterations have converged. The compressibility coefficients are calculated with $p_{a}=0 \mathrm{~Pa}$ and $p_{b}=1333.2 \mathrm{~Pa}$.

The number of coupling iterations per time step averaged over an entire simulation is given in table 1 for both the IAC method and the IBQN-LS method with a convergence tolerance of $\epsilon_{o}=10^{-3}\left\|R_{1}\right\|_{2}$. The simulation with the IAC method required least CPU time and the duration of the simulations with the IBQN-LS method relative to this simulation is also tabulated. The simulation with the IBQN-LS method is greatly accelerated by reusing information from the previous time steps but this technique becomes counterproductive when too much time steps are reused because too old information will cause errors in the leastsquares Jacobians. In this case, no more than 12 time steps should be reused but the variation near the optimum is limited.

To demonstrate that the IAC method is robust with respect to $p_{a}$ and $p_{b}$, this simulation has also been performed with $p_{a}=500 \mathrm{~Pa}$ and different values of $p_{b}$, namely $p_{b}=p_{a}+1000 \mathrm{~Pa}, p_{b}=p_{a}+100 \mathrm{~Pa}, p_{b}=p_{a}+10 \mathrm{~Pa}$ and $p_{b}=p_{a}+1 \mathrm{~Pa}$. All these simulations gave the same result and they all required on average 5 
coupling iterations per time step.

\subsection{Carotid bifurcation}

The model for the carotid artery is based on a geometry from a healthy volunteer in which an eccentric plaque in the interna was added. Starting from CT-scans, the geometry was three-dimensionally reconstructed with Mimics (Materialise NV, Leuven, Belgium). The fluid domain is discretized with 87613 cells and it has the same mesh on the fluid-structure interface as the structural model that consists of one layer with 8299 quadrilateral shell elements with 8 nodes as shown in figure 4 . The distance between the inlet at the common carotid artery and the two outlets (interna and externa) is $0.095 \mathrm{~m}$.

The same flow solver and structural solver as in the previous example have been used, with the same material properties and boundary conditions. The pressure contours on the fluid-structure interface are shown in figure 5.

The average number of iterations and the relative duration of the simulations with the IAC and IBQN-LS method are given in table 2. For these simulations with a longer and more complex domain, the difference in duration between both methods is much larger than for the straight tube because the convergence of the flow solver is significantly slower in the simulations of the bifurcation with IBQNLS. On the other hand, the difference in number of coupling iterations is similar to the simulations of the flexible tube. The number of coupling iterations in every time step is shown in figure 6.

\section{Conclusion}

Both the IAC method and the IBQN-LS can be used to simulate FSI in the geometry of a carotid bifurcation. The IAC method is faster because information about the structural solver is included within the flow solver by adding a source term in the continuity equation whereas the IBQN-LS method couples two black box solvers by approximating their Jacobians.

\section{Acknowledgments}

The authors gratefully acknowledge the funding of Joris Degroote and Peter Bruggeman by a Ph.D. fellowship of the Research Foundation - Flanders. Joris Degroote also acknowledges a grant of the Research Foundation - Flanders for a long stay abroad at the Massachusetts Institute of Technology (MIT). Abigail Swillens is 
funded by a grant of the Special Fund for Scientific Research of Ghent University (BOF).

\section{References}

[1] Quarteroni A. Cardiovascular mathematics. Proceedings of the International Congress of Mathematicians, vol. I, Sanz-Solé M, Soria J, Varona J, Verdera J (eds.), European Mathematical Society: Madrid, Spain, 2006; 479-512.

[2] Dumont K, Vierendeels J, Segers P, Van Nooten G, Verdonck P. Predicting ATS open pivot (TM) heart valve performance with computational fluid dynamics. Journal of Heart Valve Disease 2005; 14(3):393-399.

[3] Dumont K, Vierendeels J, Kaminsky R, Van Nooten G, Verdonck P, Bluestein D. Comparison of the hemodynamic and thrombogenic performance of two bileaflet mechanical heart valves using a CFD/FSI model. Journal of Biomechanical Engineering - Transactions of the ASME 2007; 129(4):558-565.

[4] dos Santos N, Gerbeau JF, Bourgat JF. Partitioned FSI strategy for simulations of a thin elastic valve. European Conference on Computational Fluid Dynamics ECCOMAS CFD 2006, Wesseling P, Oñate E, Périaux J (eds.), ECCOMAS: Delft, The Netherlands, 2006; 1-10.

[5] Taylor C, Draney M, Ku J, Parker D, Steele B, Wang K, Zarins C. Predictive medicine: Computational techniques in therapeutic decision-making. Computer Aided Surgery 1999; 4:231-247.

[6] Wilson N, Arko F, Taylor C. Predicting changes in blood flow in patientspecific operative plans for treating aortoiliac occlusive disease. Computer Aided Surgery July 2005; 10(4):257-277.

[7] Formaggia L, Gerbeau JF, Nobile F, Quarteroni A. On the coupling of 3D and 1D Navier-Stokes equations for flow problems in compliant vessels. Computer Methods in Applied Mechanics and Engineering 2001; 191:561582.

[8] Cebral J, Löhner R, Soto O, Choyke P, Yim P. Patient-Specific Simulation of Carotid Artery Stenting Using Computational Fluid Dynamics, Lecture Notes in Computer Science, vol. Medical Image Computing and ComputerAssisted Intervention, chap. 280. Springer Berlin, Heidelberg, 2001; 193219. 
[9] Gerbeau JF, Vidrascu M, Frey P. Fluid-structure interaction in blood flows on geometries based on medical imaging. Computers \& Structures 2005; 83(2-3):155-165.

[10] Hron J, Turek S. A monolithic FEM/multigrid solver for ALE formulation of fluid structure interaction with application in biomechanics. Fluid-Structure Interaction - Modelling, Simulation, Optimisation, Bungartz HJ, Schäfer M (eds.), no. 53 in Lecture Notes in Computational Science and Engineering, Springer, Berlin, 2006; 146-170. ISBN 3-540-34595-7.

[11] Causin P, Gerbeau JF, Nobile F. Added-mass effect in the design of partitioned algorithms for fluid-structure problems. Computer Methods in Applied Mechanics and Engineering 2005; 194(42-44):4506-4527.

[12] Küttler U, Wall W. Fixed-point fluid-structure interaction solvers with dynamic relaxation. Computational Mechanics DOI: 101007/s00466-0080255-5 2008; .

[13] Vierendeels J. Implicit coupling of partitioned fluid-structure interaction solvers using reduced-order models. Fluid-Structure Interaction - Modelling, Simulation, Optimisation, Bungartz HJ, Schäfer M (eds.), no. 53 in Lecture Notes in Computational Science and Engineering, Springer, Berlin, 2006; 1-18. ISBN 3-540-34595-7.

[14] Vierendeels J, Lanoye L, Degroote J, Verdonck P. Implicit coupling of partitioned fluid-structure interaction problems with reduced order models. Computers \& Structures 2007; 85(11-14):970-976.

[15] Degroote J, Bathe KJ, Vierendeels J. Performance of a new partitioned procedure versus a monolithic procedure in fluid-structure interaction. Computers \& Structures 2009; DOI:10.1016/j.compstruc.2008.11.013.

[16] Heil M. An efficient solver for the fully coupled solution of largedisplacement fluid-structure interaction problems. Computer Methods in Applied Mechanics and Engineering 2004; 193:1-23.

[17] Bathe KJ, Zhang H. Finite element developments for general fluid flows with structural interactions. International Journal for Numerical Methods in Engineering 2004; 60:213-232.

[18] Matthies H, Steindorf J. Partitioned strong coupling algorithms for fluidstructure interaction. Computers \& Structures 2003; 81:805-812. 
[19] Matthies H, Niekamp R, Steindorf J. Algorithms for strong coupling procedures. Computer Methods in Applied Mechanics and Engineering 2006; 195:2028-2049.

[20] Dettmer W, Perić D. A computational framework for fluidstructure interaction: Finite element formulation and applications. Computer Methods in Applied Mechanics and Engineering 2006; 195(41-43):5754-5779.

[21] Tezduyar T, Sathe. Modelling of fluid-structure interactions with the spacetime finite elements: Solution techniques. International Journal for Numerical Methods in Fluids 2007; 54(6-8):855-900.

[22] Tezduyar T, Sathe S, Schwaab M, Conklin B. Arterial fluid mechanics modeling with the stabilized space-time fluid-structure interaction technique. International Journal for Numerical Methods in Fluids 2008; 57(5):601-629.

[23] Peskin C. Flow patterns around heart valves: a numerical method. Journal of Computational Physics 1972; 10:252-271.

[24] Peskin C. The immersed boundary method. Acta Numerica 2002; 11:479517.

[25] van Loon R, Anderson P, de Hart J, Baaijens F. A combined fictitious domain/adaptive meshing method for fluid-structure interaction in heart valves. International Journal for Numerical Methods in Fluids 2004; 46:533-544.

[26] van Loon R, Anderson P, van de Vosse F. A fluid-structure interaction method with solid-rigid contact for heart valve dynamics. Journal of Computational Physics 2006; 217:806-823.

[27] Gerbeau JF, Vidrascu M. A quasi-Newton algorithm based on a reduced model for fluid-structure interaction problems in blood flows. ESAIM: Mathematical Modelling and Numerical Analysis 2003; 37(4):631-648.

[28] Degroote J, Bruggeman P, Haelterman R, Vierendeels J. Stability of a coupling technique for partitioned solvers in FSI applications. Computers \& Structures 2008; 86:2224-2234. DOI: 10.1016/j.compstruc.2008.05.005.

[29] Riemslagh K, Vierendeels J, Dick E. Coupling of a Navier-Stokes solver and an elastic boundary solver for unsteady problems. 4th European Computational Fluid Dynamics Conference, Papailiou K, Tsahalis D, Périaux J, Hirsch C, Pandolfi M (eds.), John Wiley \& Sons: Athens, Greece, 1998; 1040-1045. 
[30] Vierendeels J, Riemslagh K, Dick E, Verdonck P. Computer simulation of intraventricular flow and pressure gradients during diastole. Journal of Biomechanical Engineering 2000; 122:667-674.

[31] Råback P, Ruokolainen J, Lyly M, Järvinen E. Fluid-structure interaction boundary conditions by artificial compressibility. ECCOMAS Computational Fluid Dynamics Conference, ECCOMAS: Swansea, Wales, UK, 2001.

[32] Råback P, Järvinen E, Ruokolainen J. Computing the artificial compressibility field for partitioned fluid-structure interaction simulations. 8th. World Congress on Computational Mechanics, 5th. European Congress on Computational Methods in Applied Sciences and Engineering, Venice, Italy, 2008; 1 .

[33] Riemslagh K, Vierendeels J, Dick E. An efficient coupling procedure for flexible wall fluid-structure interaction. European Congress on Computational Methods in Applied Sciences and Engineering, ECCOMAS: Barcelona, Spain, 2000; 13.

[34] Vierendeels J, Riemslagh K, Dick E. Flow in moving geometries and fluidstructure interaction. Course given on 23 August 2001 during the European Mathematical Society Euro Summer School August 2001. Charles University Prague.

[35] Chorin A. A numerical method for solving incompressible viscous flow problems. Journal of Computational Physics 1967; 2:12-26.

[36] Témam R. Sur l'approximation de la solution des équations de NavierStokes par la méthode des pas fractionnaires (ii). Archive for Rational Mechanics and Analysis 1969; 33(5):377-385.

[37] Peyret R. Unsteady evolution of a horizontal jet in a stratified fluid. Journal of Fluid Mechanics 1976; 78(1):49-63.

[38] Merkle C, Athavale M. Time-accurate unsteady incompressible flow algorithm based on artificial compressibility. AIAA Paper 87-1137 1987; :397407.

[39] Botnar R, Rappitsch G, Scheidegger M, Liepsch D, Perktold K, Boesiger P. Hemodynamics in the carotid artery bifurcation: a comparison between numerical simulations and in vitro MRI measurements. Journal of Biomechanics 2000; 33:137-144. 
[40] Prosi M, Perktold K, Ding Z, Friedman M. Influence of curvature dynamics on pulsatile coronary artery flow in a realistic bifurcation model. Journal of Biomechanics 2004; 37:1767-1775.

[41] Scotti C, Finol E. Compliant biomechanics of abdominal aortic aneurysms: A fluidstructure interaction study. Computers \& Structures 2007; 85:10971113.

[42] Perktold K, Rappitsch G. Computer simulation of local blood flow and vessel mechanics in a compliant carotid artery bifurcation model. Journal of Biomechanics 1995; 28(7):845-856.

[43] Yanga X, Liu Y, Yang J. Fluid-structure interaction in a pulmonary arterial bifurcation. Journal of Biomechanics 2007; 40:2694-2699.

[44] Marzo A, Luo X, Bertram C. Three-dimensional collapse and steady flow in thick-walled flexible tubes. Journal of Fluids and Structures 2005; 20:817835 .

[45] Lesoinne M, Farhat C. Geometric conservation laws for flow problems with moving boundaries and deformable meshes and their impact on aeroelastic computations. Computer Methods in Applied Mechanics and Engineering 1996; 134:71-90.

[46] Farhat C, Lesoinne M, Le Tallec P. Load and motion transfer algorithms for fluid/structure interaction problems with non-matching discrete interfaces: momentum and energy conservation, optimal discretization and application to aeroelasticity. Computer Methods in Applied Mechanics and Engineering 1998; 157:95-114.

[47] de Boer A, van Zuijlen A, Bijl H. Review of coupling methods for nonmatching meshes. Computer Methods in Applied Mechanics and Engineering 2007; 196:1515-1525.

[48] Vierendeels J, Dumont K, Dick E, Verdonck P. Analysis and stabilization of fluid-structure interaction algorithm for rigid-body motion. AIAA Journal 2005; 43(12):2549-2557.

[49] Mok D, Wall W. Partitioned analysis schemes for the transient interaction of incompressible flows and nonlinear flexible structures. Trends in computational structural mechanics, Schweizerhof K, Wall K WA Bletzinger (eds.), CIMNE: Barcelona, 2001. 
[50] Golub GH, Loan CFV. Matrix computations. 3rd edn., Johns Hopkins University Press: Baltimore, MD, USA, 1996.

[51] Eisenstat S, Elman H, Schultz M. Variational iterative methods for nonsymmetric systems of linear equations. SIAM Journal on Numerical Analysis 1983; 20(2):345-357.

[52] Fernandez M, Moubachir M. A Newton method using exact Jacobians for solving fluid-structure coupling. Computers \& Structures 2005; 83:127-142. 


\section{$7 \quad$ Figures and tables}

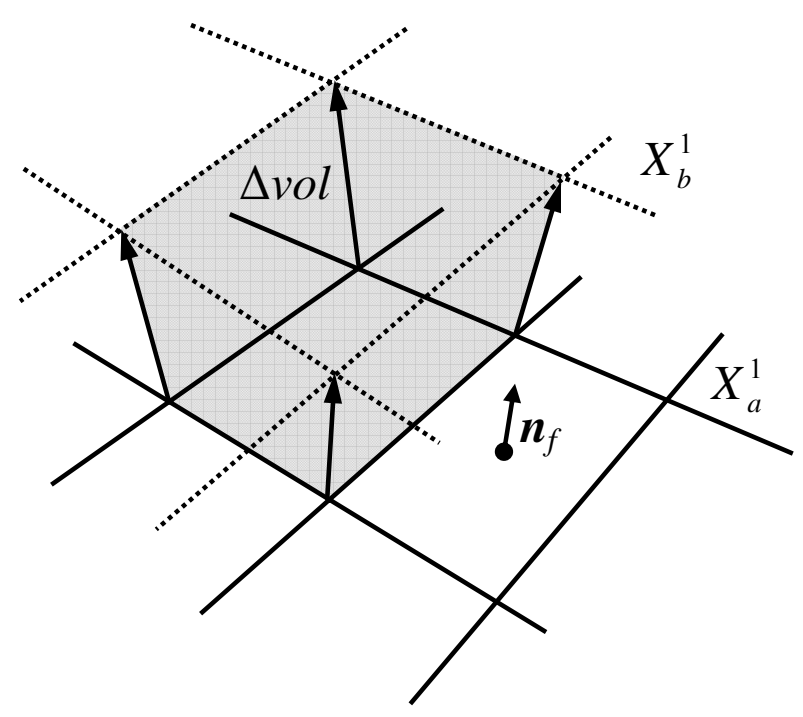

Figure 1: Volume $\Delta v o l$ swept by the interface as the latter is displaced from $X_{a}^{1}$ to $X_{b}^{1}$ with $\boldsymbol{n}_{f}$ the unit normal pointing outward of the fluid domain. 


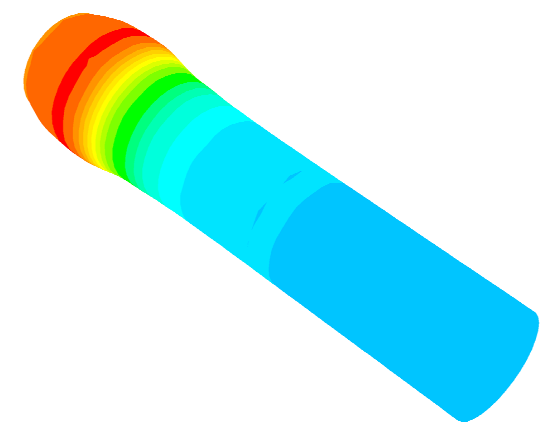

(a) $t=0.0025 \mathrm{~s}$

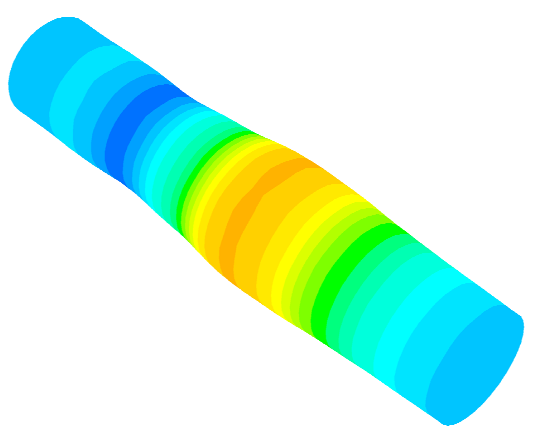

(c) $t=0.0075 \mathrm{~s}$

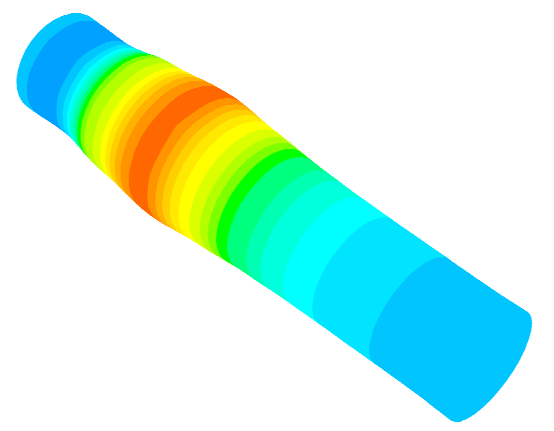

(b) $t=0.0050 \mathrm{~s}$

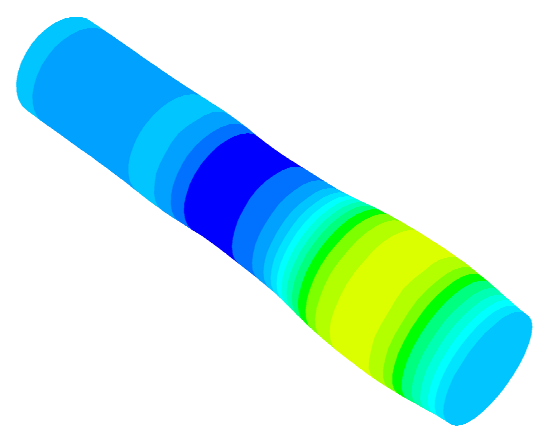

(d) $t=0.0100 \mathrm{~s}$

\begin{tabular}{|c|c|c|c|c|c|c|}
\hline$-3.00 e+02$ & $-3.00 e+01$ & $2.40 \mathrm{e}+0$ & $5.10 e+02$ & $7.80 e+02$ & $1.05 e+03$ & $1.32 \mathrm{e}+031.50 \mathrm{e}+03$ \\
\hline
\end{tabular}

Figure 2: Pressure contours on the fluid-structure interface for the simulation of the wave propagation in the straight elastic tube. The deformations have been magnified by a factor 10 . These results compare well with those presented in [52]. 


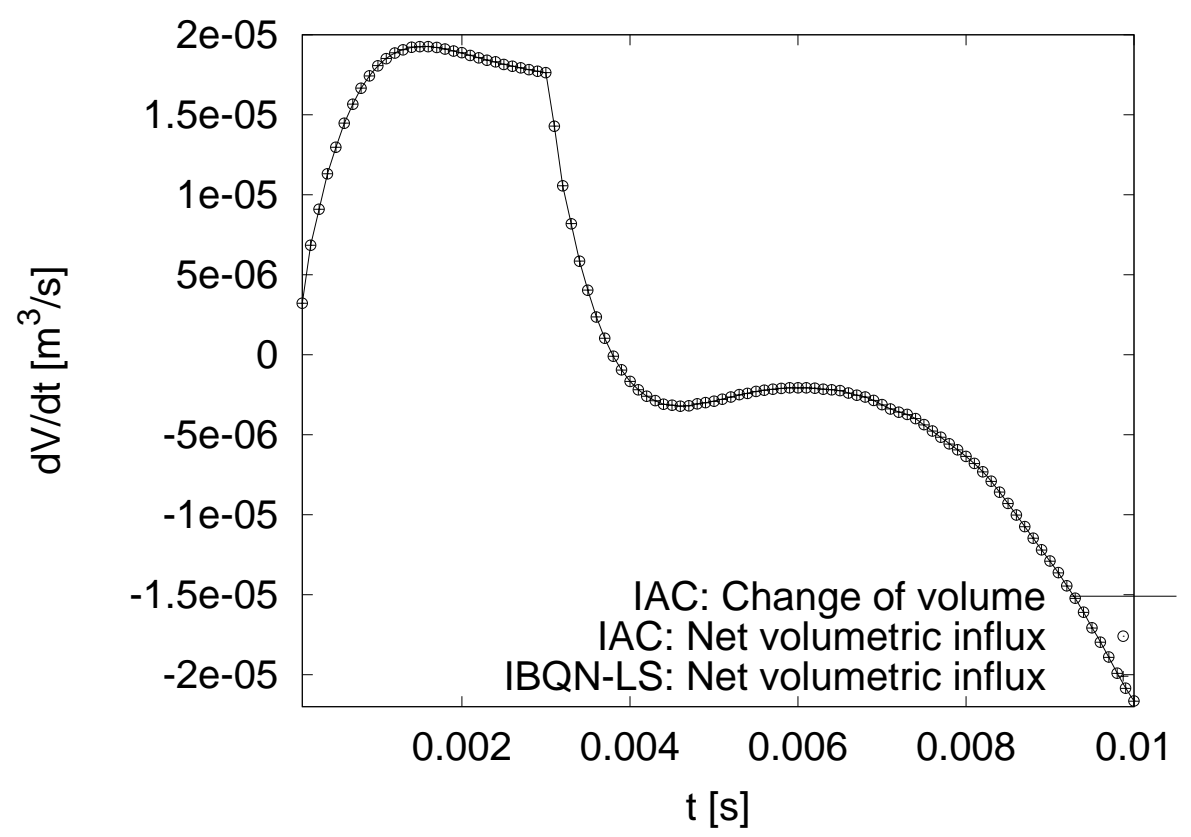

Figure 3: Comparison of the rate of change of the tube's volume $V$ and the net volumetric influx for the simulation of the wave propagation in the straight elastic tube with the IAC and IBQN-LS method. The maximal difference between the curves for the IAC method is $1.85 \cdot 10^{-9} \mathrm{~m}^{3} / \mathrm{s}$ which proves that the artificially added compressibility has disappeared once the coupling iterations have converged. 


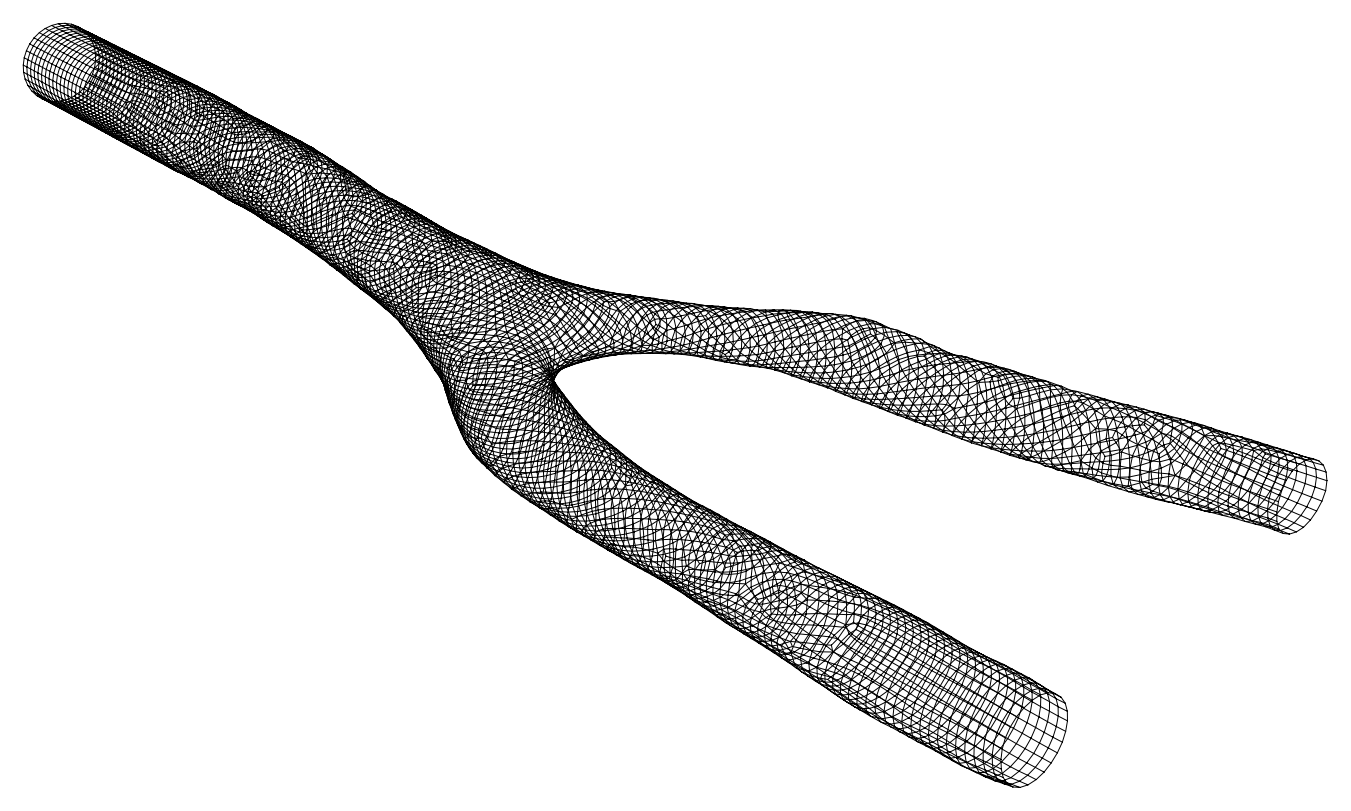

Figure 4: Mesh on the fluid-structure interface for the simulation of wave propagation in the carotid bifurcation. 


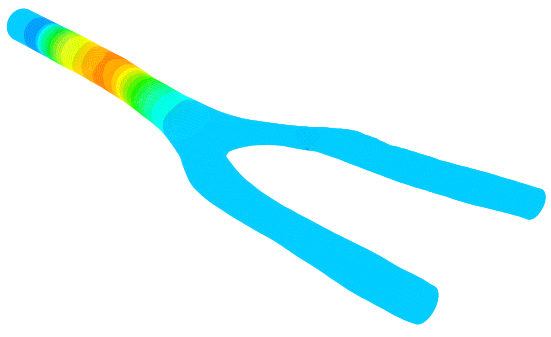

(a) $t=0.0050 \mathrm{~s}$

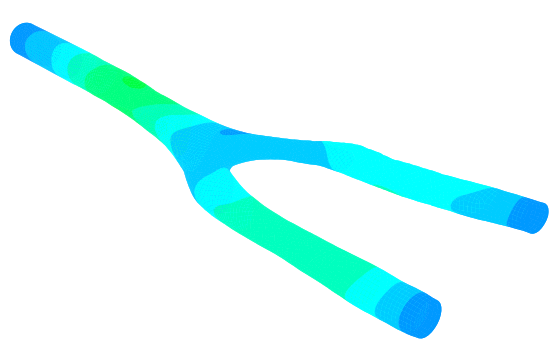

(c) $t=0.0150 \mathrm{~s}$

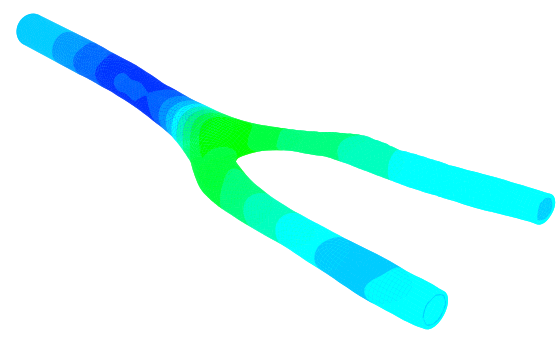

(b) $t=0.0100 \mathrm{~s}$

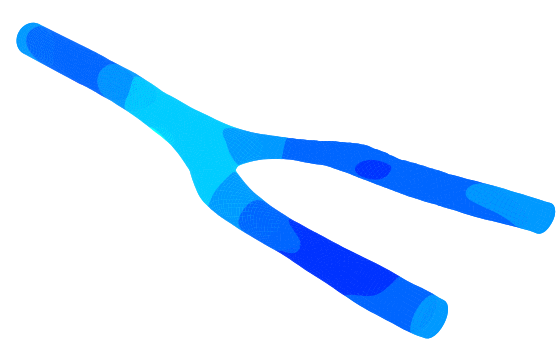

(d) $t=0.0200 \mathrm{~s}$

Figure 5: Pressure contours on the fluid-structure interface for the simulation of the wave propagation in the carotid bifurcation. 


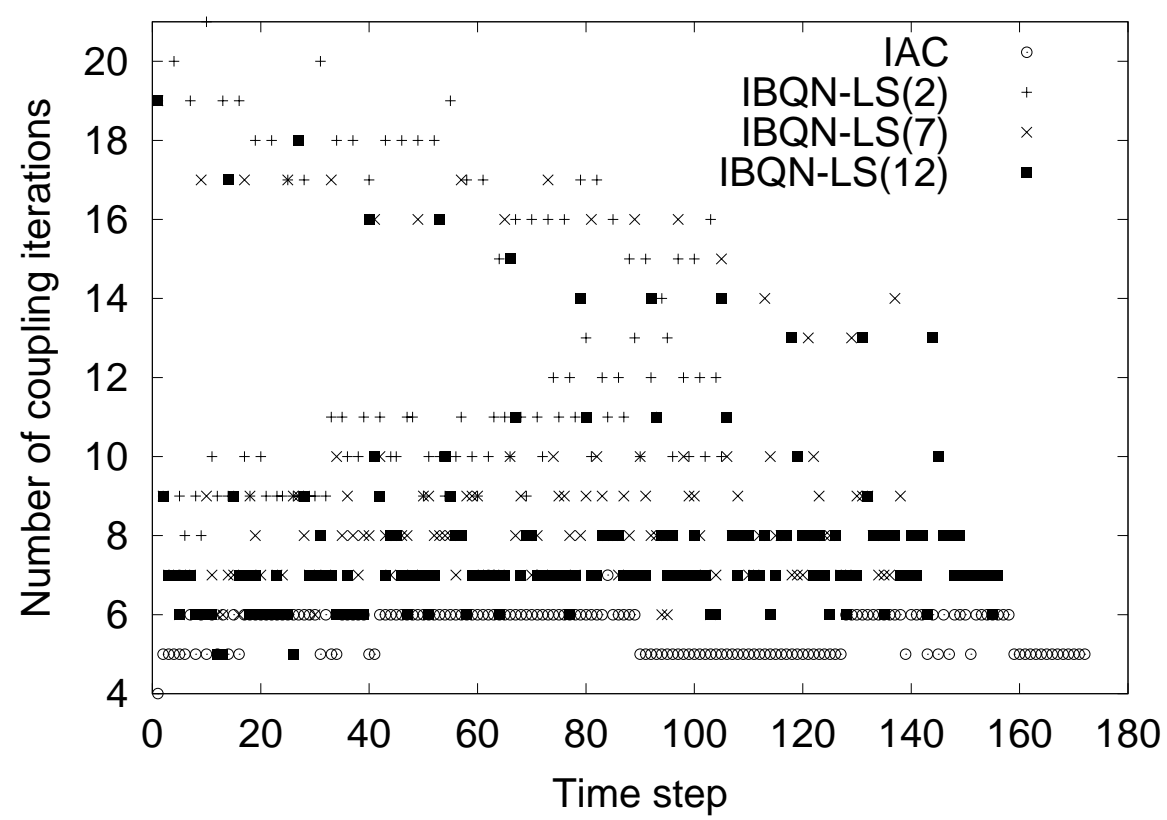

Figure 6: Number of coupling iterations per time step for the simulation of wave propagation in the carotid bifurcation. 


\begin{tabular}{|l|c|c|}
\hline Algorithm & Iterations & Duration \\
\hline IAC & 4.99 & 1.00 \\
IBQN-LS(0) & 10.48 & 2.33 \\
IBQN-LS(1) & 8.88 & 1.97 \\
IBQN-LS(5) & 7.16 & 1.57 \\
IBQN-LS(10) & 6.27 & 1.41 \\
IBQN-LS(11) & 6.16 & 1.39 \\
IBQN-LS(12) & 6.04 & 1.36 \\
IBQN-LS(13) & 6.06 & 1.37 \\
\hline
\end{tabular}

Table 1: Number of coupling iterations per time step and relative duration with respect to the IAC method (4 hours and 22 minutes) for the simulation of the wave propagation in the straight elastic tube. The number of iterations per time step has been averaged over the entire simulation. IBQN-LS $(r)$ denotes that information from $r$ time steps is reused to improve the least-squares estimation of the Jacobians. 


\begin{tabular}{|l|c|c|}
\hline Algorithm & Iterations & Duration \\
\hline IAC & 5.58 & 1.00 \\
IBQN-LS(2) & 12.56 & 5.68 \\
IBQN-LS(7) & 8.94 & 3.96 \\
IBQN-LS(12) & 7.87 & 3.45 \\
\hline
\end{tabular}

Table 2: Number of coupling iterations per time step and relative duration with respect to the IAC method ( 2 days, 11 hours and 46 minutes) for the simulation of the wave propagation in the carotid bifurcation. The number of iterations per time step has been averaged over the entire simulation. IBQN-LS $r r)$ denotes that information from $r$ time steps is reused to improve the least-squares estimation of the Jacobians. 